\title{
Terapia electroconvulsiva: perspectiva de pacientes $y$ familiares
}

\author{
Alejandra Sofía Quesada Hincapié,* Ángela Rocío Acero González ${ }^{*}$ \\ * Estudiante del semestre XIV. \\ ¥ M.D., MMF, PhD., Profesora asistente.
}

Facultad de Medicina de la Universidad de La Sabana. Colombia.

\section{RESUMEN}

Objetivo: Explorar información de los últimos 10 años sobre la percepción de pacientes y familiares en cuanto a la terapia electroconvulsiva en conocimiento, satisfacción y actitud. Material y métodos: Se realiza una revisión narrativa en bases de datos electrónicas con palabras clave en términos MeSH y búsqueda en "bola de nieve". Resultados: Se incluyeron 11 artículos. Los pacientes y familiares en su mayoría tenían conocimientos básicos de esta terapia. En general presentan una actitud positiva, siendo mejor en quienes tuvieron la experiencia en comparación con quienes no la tuvieron; estos últimos mostraron una actitud predominantemente ambivalente. Asimismo, se evidenció que quienes recibían información de sus médicos, tenían mejor actitud que quienes la obtenían por otros medios. Respecto a la satisfacción, ésta es 50\% alta en familiares y pacientes; sin embargo, para algunos la anestesia y el tratamiento generaron temor y la alteración de la memoria fue el efecto adverso más relevante. En general, consideran la terapia como un proceso benéfico para el paciente y su patología. Conclusiones: Aunque la percepción general es positiva, se evidencian múltiples aspectos por mejorar que tendrían un efecto positivo en la percepción y podrían disminuir las barreras de acceso al uso de una terapia que ha mostrado ser efectiva.

Palabras clave: Terapia electroconvulsiva, pacientes, familia, conocimiento, actitud, prácticas en salud.

\begin{abstract}
Objective: To explore information from the last ten years on patients' and families' perception of electroconvulsive therapy in knowledge, satisfaction and attitudes. Material and methods: A narrative review is performed on electronic databases with keywords in MeSH terms and snowball search. Results: Eleven articles were included. Most patients and families had a basic understanding of this therapy. In general, they present a positive attitude, being better in those who had the experience, compared to those who did not, having the latter a predominantly ambivalent attitude. Additionally, it was evident that those who received information from their doctors had a better attitude than those who obtained it by other means. Satisfaction is higher than $50 \%$ for both family members and patients, however, for some anesthesia and treatment generated fear, and memory impairment was the most relevant adverse effect. In general, they consider therapy as a beneficial process for the patient and his or her pathology. Conclusions: Although the general perception is positive, there are multiple aspects to be improved; that would have a positive effect on the perception and could decrease the barriers to access the use of a therapy that has been shown to be effective.
\end{abstract}

Keywords: Electroconoulsive therapy, patients, family, health knowledge, attitudes, health practices.

\section{INTRODUCCIÓN}

La terapia electroconvulsiva (TEC) ha sido y sigue siendo un tratamiento controvertido. ${ }^{1,2} \mathrm{~A}$ pesar de considerarse un tratamiento eficaz y seguro por los expertos, ${ }^{3}$ existen múltiples ideas sobre en qué consiste, cómo funciona y qué efectos adversos (EA) conlleva. ${ }^{4-6}$ Los medios de comunicación han difundido información

\footnotetext{
Abreviaturas:

$\mathrm{EA}=$ Efectos adversos.

TEC $=$ Terapia electroconvulsiva.
}

que concibe la terapia como un evento violento, horrible e incluso irrespetuoso de la dignidad humana, lo que deriva en la concepción negativa perpetuada en el imaginario colectivo. ${ }^{7-9}$

Este dilema estuvo en auge en 2019 igual que hace 20 años, incluyendo la creación de organizaciones (que incluso contienen profesionales de la salud) que velan por la descontinuación del tratamiento y suspensión de las terapias en curso..$^{8-12}$

Se han realizado múltiples estudios sobre la percepción de profesionales de la salud como enfermeras y médicos así como estudiantes de medicina y público general. ${ }^{9-11,13,14} \mathrm{Sin}$ embargo, la percepción de quie- 
nes viven la TEC no ha sido tan evaluada, a pesar del efecto que ésta puede producir en su aceptación y uso en la práctica clínica. ${ }^{15}$

La Real Academia de la Lengua Española (RAE) define percepción como una "sensación interior que resulta de una impresión material hecha en nuestros sentidos" o como un "conocimiento, idea" ${ }^{16}$ En el área de la salud se relacionan con el concepto: conocimiento, experiencia y actitud. Como tal, se considera que para definir la percepción de alguien sobre la terapia electroconvulsiva debemos hablar de la impresión que le genera, es decir, de la satisfacción o actitud hacia ella, e igualmente acerca del conocimiento que tiene sobre el tema. ${ }^{17,18}$

Por lo anterior, y teniendo en cuenta además que la terapia electroconvulsiva y su técnica han cambiado con el tiempo, se estima importante realizar una recopilación de datos de investigaciones respecto de la perspectiva (conocimiento, actitud y satisfacción) de la TEC por parte de los pacientes en salud mental y sus familiares que esté actualizada y que permita renovar el conocimiento acerca de estas percepciones y establecer elementos necesarios a investigar o intervenir para disminuir las barreras de acceso.

\section{Material y Métodos}

Se realiza una revisión narrativa de la literatura por medio de búsquedas en PubMed, LILACS, Ovid y ScienceDirect utilizando múltiples combinaciones de los términos MeSH: Electroconvulsive Therapy; knowledge, attitudes, practice; patients; family; y convulsive therapy; además se realizó búsqueda con el sistema "bola de nieve". Dentro de los criterios para escoger los artículos se decidió tomar los publicados en el período 2009-2019, publicaciones en inglés y en español; no obstante, se mantuvo la flexibilidad en los tipos de diseño. Se excluyeron las opiniones individuales al igual que los estudios sobre estos aspectos en personas diferentes a familias y usuarios (donde no estuviesen incluidos usuarios o familiares).

Con la finalidad de realizar las búsquedas y análisis se entiende conocimiento como la información o "conocimientos [...] que pertenecen a temas relacionados con la salud, como los procesos patológicos o las enfermedades, su prevención y tratamiento". ${ }^{19}$ Satisfacción se define como "el grado en que el individuo considera que el servicio o producto de atención

Tabla 1: Descripción de conocimientos.

\begin{tabular}{ll}
\hline Artículos & Conocimiento de pacientes \\
\hline Flamarique, 2015 & $>50 \%$ de pacientes que recibieron TEC y que no la recibieron, refieren que hay un “uso de corriente eléctrica \\
& para producir convulsión” \\
Dan, 2014 & Ningún evaluado con conocimiento completo \\
& Familiares con mayor conocimiento que pacientes, pero sin ser significativo estadísticamente \\
& Mayoría desconocía aspectos básicos, consentimiento, indicaciones, efectividad y EA \\
& No hay diferencia entre pacientes que recibieron TEC vs. quienes no recibieron TEC en pacientes o familia- \\
& res \\
Malekian, 2009 & Poco conocimiento previo \\
& Conocimiento posterior a TEC aumenta en elementos técnicos y EA \\
Grover, 2011 & Mayoría de familiares de ambos grupos conocían aspectos básicos \\
& Familiares de pacientes que recibieron TEC mejor informados \\
Rajagopal, 2012 & Ningún paciente tenía conocimiento completo \\
& Cercano a todo correcto 16\% \\
& Rudimentos del procedimiento 52-71\% \\
& Familiares 52-96\% conocimiento de varios aspectos \\
& Familiares más conocimiento \\
& Preeducación: 23.4/32 (73\%) \\
& Posteducación: 30.4/32 (95\%)
\end{tabular}


Tabla 2: Descripción de la actitud.

\begin{tabular}{|c|c|}
\hline Artículos & Actitud en pacientes y familiares \\
\hline Flamarique, 2015 & $\begin{array}{l}\text { Mayoría de los pacientes que recibieron TEC, actitud positiva } \\
\text { Pacientes que no recibieron TEC, actitud ambivalente } \\
\text { Tres pacientes que se informaron por los medios tenían una visión negativa, de éstos, uno consideró que la } \\
\text { terapia debería ser ilegal }\end{array}$ \\
\hline Dan, 2014 & $\begin{array}{l}\text { Actitud positiva } 45 \% \text { en pacientes y } 53 \% \text { en familiares } \\
\text { Actitud negativa en pacientes } 38 \% \text { y } 33 \% \text { en familiares } \\
\text { Actitud más positiva en pacientes y familiares que aprenden a partir de doctores vs. medios }\end{array}$ \\
\hline Malekian, 2009 & $\begin{array}{l}\text { Actitud positiva en general previo a la terapia } \\
\text { Mejoría de la actitud posterior al uso de la TEC }\end{array}$ \\
\hline Zhang, 2018 & $\begin{array}{l}\text { Mayoría de pacientes y familiares participantes presentaron una actitud positiva } \\
\text { Sin diferencia significativa entre pacientes y familiares }\end{array}$ \\
\hline Grover, 2011 & $\begin{array}{l}\text { Actitud positiva en familiares de pacientes que recibieron TEC ( } 0-6 \% \text { actitud negativa) } \\
\text { Grupo que recibió TEC, actitud más positiva que grupo que no recibió TEC } \\
\text { 17-55\% de los familiares de pacientes que no recibieron TEC inseguros o ambivalentes respecto a su actitud } \\
\text { Participantes informados por los médicos fueron más positivos }\end{array}$ \\
\hline Bloch, 2017 & $\begin{array}{l}\text { 90\% de adultos que recibieron TEC y } 69 \% \text { de adolescentes que recibieron TEC la consideraron efectiva } \\
\text { Actitud negativa por parte de una minoría de los pacientes que recibieron TEC (0-14\%) } \\
\text { Pacientes que no recibieron TEC: actitud más negativa }\end{array}$ \\
\hline Kheiri, 2011 & $\begin{array}{l}\text { Preeducación: 33.4/70 (47.7\%) } \\
\text { Posteducación: } 41.1 / 70 \text { (59\%) }\end{array}$ \\
\hline
\end{tabular}

médica o la manera en que lo entrega el proveedor es útil, efectivo o beneficioso". ${ }^{20} \mathrm{Y}$ la actitud como "una predisposición duradera y aprendida a comportarse de manera consistente hacia una clase dada de objetos, o un estado mental y/o neuronal persistente de preparación para reaccionar ante cierta clase de objetos no como son, sino como son concebidos para ser." ${ }^{21}$

\section{Resultados}

En las bases de datos seleccionadas se encontró un total de 32 artículos al respecto del tema a tratar. Tras la revisión de los resúmenes se excluyeron las opiniones individuales al igual que los estudios sobre opiniones del público o de estudiantes o profesionales en áreas de la salud, a partir de lo cual se encontró un total de 15 artículos. Posteriormente, se hizo una revisión completa del contenido encontrando cuatro artículos que provenían del mismo estudio y presentaban los mismos resultados. En total se obtuvieron 11 artículos; uno de ellos es un artículo de revisión y los otros 10 ofrecen información cuantitativa al respecto del tema. En cuatro artículos los autores hacen explícito el uso modificado de la TEC. En los otros no se tiene esta información.

\section{Conocimiento de pacientes y familiares}

El reporte del conocimiento sobre la terapia se estudió en seis artículos ${ }^{22-27}$ (Tabla 1), dos de ellos tratan exclusivamente el conocimiento en familiares. ${ }^{26,27}$ Tres revelan un conocimiento básico o de los rudimentos de la terapia electroconvulsiva en la mayoría de los participantes, con conocimiento menor de aspectos más específicos. ${ }^{23,24,26}$ Sin embargo, sólo en uno explican referirse al "paso de corriente eléctrica" como conocimiento básico. ${ }^{24}$ Otros dos estudios reportan que ninguno de los entrevistados mostró un conocimiento completo con respecto a la terapia. ${ }^{23,25}$ Dos estudios que realizan un contraste del conocimiento de los entrevistados antes y después de la terapia o en grupos que han recibido y que no han recibido 
TEC, muestran una clara diferencia a favor de quienes sí la recibieron y del conocimiento posterior al tratamiento; ${ }^{22,26}$ un artículo que evalúa el conocimiento antes y después de una intervención educativa en familiares de pacientes de salud mental ${ }^{27}$ demuestra un aumento drástico en su conocimiento posterior a la intervención. Es necesario advertir que en el estudio en el que la fuente principal de información para pacientes y familiares fue el médico tratante, ${ }^{23}$ el conocimiento reportado fue mayor (52-96\% de familiares tenía conocimiento de "varios" aspectos y $38-49 \%$ de los pacientes "conocimientos sobre consentimiento, mecanismo, indicaciones y efectos adversos"), lo que parece estar de acuerdo con lo previamente sustentado.

Una de las investigaciones que valora los Conocimientos de pacientes que recibieron TEC con sus familiares versus pacientes que no han sido tratados con la terapia y sus familiares, ${ }^{25}$ no muestra diferencia respecto al conocimiento de ambos

Tabla 3: Descripción de satisfacción de pacientes.

\begin{tabular}{|c|c|}
\hline Artículos & Satisfacción pacientes \\
\hline Rajagopal, 2013 & $\begin{array}{l}54 \% \text { de pacientes satisfechos con TEC en general } \\
64 \% \text { satisfechos específicamente con los resultados } \\
72 \% \text { lo volverían a hacer si lo necesitaran } \\
30 \% \text { refirieron tener miedo a la terapia } \\
\text { Insatisfacción con la información recibida fue }>30 \%\end{array}$ \\
\hline Flamarique, 2015 & $\begin{array}{l}\text { 89.5\% de los adolescentes tratados con TEC reportaron que se sintieron mejor posterior al tratamiento } \\
\text { > 50\% recibirían TEC si tuvieran que hacerlo (en grupo con y sin TEC) } \\
\text { 31.6\% estaban muy asustados, } 26.3 \% \text { algo asustados previo a la realización de la TEC } \\
\text { 63.2\% de los pacientes que recibieron TEC consideraron que era seguro } \\
\text { Los pacientes que no recibieron TEC consideraron no saber si era seguro en } 81 \% \\
\text { 57.6\% de los pacientes que recibieron TEC no están seguros de recibir adecuada información previa } \\
\text { Los pacientes que no recibieron TEC consideraron no saber o tener una información clara acerca } \\
\text { del procedimiento }\end{array}$ \\
\hline Brown, 2018 & $\begin{array}{l}\text { 85.5\% de los participantes refirieron un beneficio significativo } \\
\text { 98.7\% de los participantes consideraron que era un tratamiento seguro } \\
97.4 \% \text { de los participantes lo volverían a hacer si fuera necesario }\end{array}$ \\
\hline Malekian, 2009 & $\begin{array}{l}\text { Los pacientes lo consideraron beneficioso en } 59 \% \\
\text { Los pacientes lo consideraron satisfactorio en } 79.5 \% \\
90 \% \text { de los pacientes consideraron que la información sobre riesgos fue inadecuada } \\
>70 \% \text { de pacientes y familiares consideraron que no recibieron adecuada información (68.2-95.5\%) }\end{array}$ \\
\hline Zhang, 2018 & $\begin{array}{l}54.2 \% \text { volverían a realizarla si fuera necesario } \\
31.5 \% \text { tenían preocupaciones } \\
56 \% \text { de los pacientes consideraron que recibieron información adecuada } \\
65.4 \% \text { de los pacientes la consideraron segura }\end{array}$ \\
\hline Rajagopal, 2012 & $\begin{array}{l}\text { 60-66\% de los participantes lo consideraron beneficioso } \\
66 \% \text { volvería a realizarla si fuera necesario } \\
62 \% \text { lo recomendaría a otros } \\
22-40 \% \text { reportaron tener incomodidad con miedo al tratamiento, tratamiento retrasado y EA } \\
64 \% \text { estuvo disgustado con información previa } \\
58-68 \% \text { tuvieron dudas respecto a la satisfacción con el proceso del consentimiento }\end{array}$ \\
\hline Bloch, 2017 & $\begin{array}{l}\text { "Más o menos la mitad" de los participantes consideraron que generó una contribución significativa } \\
\text { con su terapia }\end{array}$ \\
\hline
\end{tabular}


Tabla 4: Descripción de satisfacción en familiares.

\begin{tabular}{|c|c|}
\hline Artículo & Satisfacción de familiares \\
\hline $\begin{array}{l}\text { Rajagopal, } \\
2013\end{array}$ & $\begin{array}{l}\text { Familiares más satisfechos que pacientes } \\
70-100 \% \text { satisfechos en general } \\
30 \% \text { miedo al tratamiento }\end{array}$ \\
\hline $\begin{array}{l}\text { Malekian, } \\
2009\end{array}$ & $\begin{array}{l}90.9 \% \text { consideraron que el tratamiento } \\
\text { fue satisfactorio } \\
\text { Familiares más satisfechos con el trata- } \\
\text { miento } \\
72.7 \% \text { beneficioso } \\
>70 \% \text { de pacientes y familiares conside- } \\
\text { ran que no recibieron adecuada informa- } \\
\text { ción ( } 45.5-81.8 \% \text { ) } \\
\text { Familiares más satisfechos con informa- } \\
\text { ción }\end{array}$ \\
\hline $\begin{array}{l}\text { Zhang, } \\
2018\end{array}$ & $\begin{array}{l}70.4 \% \text { volverían a realizarla si fuera } \\
\text { necesario } \\
75 \% \text { adecuada información } \\
\text { Familiares más satisfechos con informa- } \\
\text { ción }\end{array}$ \\
\hline $\begin{array}{l}\text { Rajagopal, } \\
2012\end{array}$ & $\begin{array}{l}\text { Familiares más satisfechos ( } 66-99 \%) \\
93 \% \text { felices de que el paciente lo reci- } \\
\text { biera } \\
\text { 94\% lo recomendarían a otros } \\
\text { 34\% miedo a la TEC }\end{array}$ \\
\hline
\end{tabular}

grupos, donde los dos en su mayoría desconocen aspectos básicos de la terapia así como respecto al consentimiento informado, EA, indicaciones o efectividad. Esto puede deberse a que la mayoría de los participantes de este estudio obtuvieron la información a partir de los medios de comunicación (televisión, periódicos, etc.), lo que puede llevar a malas interpretaciones y desconocimiento en general. ${ }^{25}$

Dos estudios reportan mayor conocimiento por parte de los familiares, ${ }^{23,25}$ los autores explican que esto es debido a que los familiares suelen tener mejor acceso a diversas fuentes de información ${ }^{25}$ y las alteraciones en la memoria a causa de la patología tratada o al procedimiento. ${ }^{18}$

\section{Actitud en pacientes y familiares}

La actitud de pacientes y familiares se observó en siete estudios (Tabla 2). ${ }^{22,24-29}$ La mayoría de artículos mos- traron una actitud positiva hacia la terapia en pacientes que la recibieron y sus familiares. ${ }^{22,24,26,28,29}$

En tres artículos ${ }^{24,26,28}$ donde se hace una comparación de familiares y pacientes que recibieron TEC versus un grupo que no siguió terapia, se observa que la actitud tiene una tendencia a ser más positiva en los grupos que tuvieron la experiencia. Además, dos de ellos ${ }^{24,26}$ muestran que quienes no conocen de primera mano la técnica, presentaron una actitud ambivalente. Esto podría sugerir que consideran no tener información suficiente para definir una actitud ante la TEC.

En contraposición a lo previamente descrito un estudio revela una visión positiva en apenas 45\% de los pacientes y $53 \%$ de los familiares; ${ }^{25}$ el autor concluye que esto puede deberse a que la mayoría de los participantes del mismo no recibieron terapia electroconvulsiva, asociado además a que la fuente principal de información reportada fueron los medios

Tabla 5: Descripción de efectos adversos.

\begin{tabular}{|c|c|}
\hline Artículo & Efectos adversos \\
\hline $\begin{array}{l}\text { Flamarique, } \\
2015\end{array}$ & $\begin{array}{l}55.6 \% \text { reportaron EA } \\
\text { Confusión }(47.4 \%) \\
\text { Alteraciones en la memoria }(42.1 \%) \\
\text { Cefalea }(21.1 \%) \\
\text { Náusea }(15.8 \%)\end{array}$ \\
\hline $\begin{array}{l}\text { Brown, } \\
2018\end{array}$ & $\begin{array}{l}\text { Síntomas adversos antes y después de } \\
\text { TEC: } \\
\text { Único aumento: alteraciones en la memo- } \\
\text { ria a corto plazo (sin diferencia estadísti- } \\
\text { camente significativa) } \\
\text { Los otros síntomas disminuyeron: } \\
\text { dolor muscular, confusión, dificultad para } \\
\text { la concentración }\end{array}$ \\
\hline $\begin{array}{l}\text { Malekian, } \\
2009\end{array}$ & $\begin{array}{l}\text { Principalmente pérdida de memoria } \\
(95.5 \%) \\
\text { Vértigo } 59 \% \text {, mialgia } 40 \% \text { y vómito } 18 \%\end{array}$ \\
\hline $\begin{array}{l}\text { Zhang, } \\
2018\end{array}$ & $\begin{array}{l}\text { 57\% reportan EA: } \\
\text { Pérdida de memoria } 62.6 \% \\
\text { Cefalea } 33.6 \% \\
\text { Mialgias } 29.9 \%\end{array}$ \\
\hline $\begin{array}{l}\text { Bloch, } \\
2017\end{array}$ & $\begin{array}{l}\text { En orden de aparición reportan: } \\
\text { 1. Problemas de memoria } \\
\text { 2. Confusión } \\
\text { 3. Fatiga }\end{array}$ \\
\hline
\end{tabular}


de comunicación y, como se encontró en éste y otros dos artículos, al comparar otras fuentes versus quienes recibieron información de los médicos, los segundos presentan mejor actitud. ${ }^{24-26}$

Un estudio realizó una intervención educativa en familiares de pacientes de salud mental y usó una medición previa y posterior, ${ }^{27}$ esta investigación reveló una mejoría significativa en la actitud. Lo anterior asociado con los demás hallazgos puede dirigirnos a pensar que cuanto mayor y más acertada sea la información adquirida por pacientes y familiares, más positiva será entonces la visión ante la TEC.

\section{Satisfacción y experiencia con la TEC}

\section{Pacientes}

Los detalles de la satisfacción en pacientes fueron abordados en siete artículos (Tabla 3). ${ }^{17,22,23,28-30} \mathrm{La}$ satisfacción, efectividad y/o el beneficio percibido fueron los hallazgos preponderantes, así como la posibilidad de volver a realizar la terapia de ser necesario o de recomendarla a otros, con porcentajes entre 54$90 \% .{ }^{17,22-24,28-30}$ De igual manera, la mayoría lo consideraron un procedimiento seguro (65.4-98.4\%). ${ }^{24,29,30}$

Sin embargo, también hubo un reporte de temor al tratamiento, con una fracción de aproximadamente $1 / 3$ de los participantes de los estudios, ${ }^{17,23,24,29}$ y se presenta principalmente asociado a la inyección de anestesia ${ }^{24} \mathrm{o}$ al uso de electricidad. ${ }^{29}$

La percepción de los pacientes de la información dada previo al tratamiento fue negativa en los cinco artículos que la reportaron (> 30-> 70\%). ${ }^{17,22-24,29}$ Asimismo, un porcentaje de pacientes $y / 0$ familiares (23-77\%) consideró aceptar el procedimiento porque podría realizarse aun sin su consentimiento, $22,25 \mathrm{O}$ porque la TEC era una "cura" para su enfermedad (53-95.5\%), ${ }^{22,25}$ incluso algunos decidieron acceder por su confianza en el médico (20-100\%). ${ }^{22,23}$

\section{Familiares y pacientes}

De los 11 artículos obtenidos cuatro evaluaron además la satisfacción de los familiares (Tabla 4). ${ }^{17,22,23,29}$ Los resultados muestran un alto porcentaje de satisfacción con el tratamiento (66-100\%), el cual es mayor en comparación con el de los pacientes. De igual forma, los familiares reportaron estar más satisfechos con la información recibida. Los autores relacionan esto con el hecho de que los familiares no tienen la experiencia directa con la enfermedad, la terapia o EA secundarios a ésta, asimismo son los familiares quienes generalmente deben firmar el consentimiento informado y por tanto, podrían recibir mejor información respecto al proceso. ${ }^{22}$ En dos de estos artículos se muestra también en los familiares la existencia de miedo ante la realización del procedimiento. ${ }^{17,23}$

\section{Efectos adversos}

Cinco artículos evaluaron los EA referidos posterior a la terapia (Tabla 5). ${ }^{22,24,28-30}$ En ellos se reportan pérdida de memoria y confusión como los EA más comunes, aunque también se describen mialgias, cefalea, vértigos y fatiga.

Uno de estos estudios ${ }^{30}$ aplica cuestionarios antes y después de la terapia sobre EA en los pacientes, teniendo en cuenta que los EA asociados con la terapia suelen encontrarse en depresión mayor. Los resultados identifican como único efecto adverso que los pacientes reportan, posterior a la terapia, pérdida de memoria a corto plazo sin que sea un aumento estadísticamente significativo.

\section{Discusión}

A pesar de la evidencia que demuestra que la TEC es un tratamiento eficaz y seguro, ${ }^{3,31}$ su uso viene en disminución ${ }^{32,33}$ y la percepción de quienes podrían usarlo a futuro se mostró ambivalente o negativa, como se había descrito en algunos estudios previos. ${ }^{6,24,26} \mathrm{El}$ objetivo de esta revisión fue recopilar y analizar la información registrada en los últimos 10 años con respecto a la percepción de los involucrados directamente en el tratamiento y con la TEC.

Los resultados muestran que el conocimiento de pacientes y familiares, en la mayoría de los casos, es básico, ${ }^{23,24,26}$ y posterior a una intervención o a la realización de la terapia se presenta una mejoría significativa. ${ }^{22,26,27}$ Se evidencia además que existe una diferencia significativa entre quienes recibieron información de un médico tratante o psiquiatra versus quienes la recibieron por otros medios. ${ }^{25-27}$ Esto concuerda con lo encontrado en otros estudios y sugiere que existe una información insuficiente previa al tratamiento, la cual mejora posterior a una intervención o a la experiencia propia. $1,5,18,34,35$

Con respecto a la actitud, ésta fue predominantemente positiva, ${ }^{22,24,26,28,29}$ hallazgo que también ha sido descrito por otros autores previamente. ${ }^{18,35,36}$ Cabe resaltar que esta actitud muestra ser más 
positiva en pacientes y familiares que estuvieron expuestos al tratamiento, así como en quienes tuvieron como fuente de información a su médico tratante, ${ }^{24-26,28}$ y contrasta con la actitud del público en general. ${ }^{4,8,9}$ Se ha postulado que la información desempeña un papel importante en la actitud, lo que va en la misma línea del estudio de Kheiri, ${ }^{27}$ en el que posterior a una intervención educativa la actitud fue significativamente más positiva. Otros estudios también revelan que a mayor información y experiencia mejor percepción. ${ }^{9}$

La mayoría de los participantes expresaron satisfacción, sensación de beneficio y efectividad. Estos datos son similares a los encontrados en artículos anteriores, ${ }^{15,18,26,36-38}$ incluyendo la revisión realizada en 2010, los cuales sugieren además que la efectividad mostrada por la terapia ${ }^{31,32}$ se correlaciona con lo referido por los pacientes. Sin embargo, también es usual el reporte de miedo en pacientes y familiares; en esta revisión es alusivo sobre todo ante a la inyección de anestesia ${ }^{24}$ o el uso de electricidad, ${ }^{29}$ hallazgos que también han sido previamente descritos por algunos autores. ${ }^{15,39,40}$ Asimismo, al indagar la percepción de la información brindada, los pacientes y familiares la consideraron negativa en la mayoría de los casos, hallazgo que fue descrito igualmente en los resultados de la revisión y de otros estudios previos. $5,18,34,35$

En lo que concierne a la satisfacción en familiares se observó que ésta fue mayor que en pacientes, incluyendo la relacionada con la información recibida. ${ }^{17,22,23,29}$ Lo anterior es congruente con la revisión de 2010 y otros estudios previos ${ }^{18,36,38}$ y se considera que es posible que sea secundario a que los familiares no son quienes viven la enfermedad o son expuestos a la terapia y por ende, no viven sus reveses, e igualmente por ser ellos quienes deben firmar el consentimiento informado, suelen estar provistos de mayor cantidad de información para su aceptación. ${ }^{22,36,38}$

Las razones para aceptar el procedimiento son diversas y no necesariamente relacionadas con la enfermedad o con los efectos reales de la terapia, sino más bien con la creencia de que es una cura, ${ }^{22,25}$ aceptación por confianza en el médico ${ }^{22,23}$ o a la aceptación pasiva por considerar que podría realizarse aun sin su consentimiento; ${ }^{22,25}$ estas razones han sido también descritas previamente $5,34,38,39$ y requieren una revisión más profunda.

En cuanto a los EA, se reportan como principales las alteraciones de memoria y la confusión, lo que concuerda con lo investigado previamente. ${ }^{18,39,40} \mathrm{Sin}$ embargo, un artículo ${ }^{30}$ que hace una comparación previa y posterior de la TEC muestra como único síntoma el aumento de la pérdida de memoria a corto plazo, el cual no es estadísticamente significativo. Aunque otros estudios son concordantes con este hallazgo, ${ }^{15,41}$ este tema requiere más investigación.

En resumen, la consistencia en algunos de estos hallazgos podría ser una buena base para tomar determinaciones en el futuro encaminadas a abordar los hechos que se pudieran considerar como negativos entre los que viven la experiencia de la TEC (la información recibida, la firma del consentimiento informado, la incidencia y naturaleza de los EA, miedos a las implicaciones del procedimiento, etc.) con el fin de optimizar el proceso del tratamiento y por ende, la experiencia y satisfacción con el mismo.

Durante el desarrollo de esta revisión se evidenció que existen múltiples limitaciones en los estudios, la falta de métodos de evaluación validados que permitan unificar lo que se debe considerar como el conocimiento mínimo de pacientes y familiares, encuestas cerradas que dificultan la profundización en opiniones, así como muestras reducidas de participantes en los estudios; dichas limitaciones derivan en mayor dificultad para la adecuada inducción a partir de los resultados. Es importante recalcar la necesidad de generar más investigación con respecto a la perspectiva de pacientes y familiares, pues los resultados no han sido suficientes. De la misma manera, en busca de una mayor profundidad de los datos se considera necesario utilizar métodos de investigación cualitativos que puedan brindar una visión más completa sobre esta vivencia.

\section{Conclusiones}

Los hallazgos de esta revisión evidencian un panorama similar en la percepción de pacientes y familiares al encontrado hace 10 años, con una percepción en general más positiva que otros grupos (población general, profesionales de la salud, estudiantes de medicina), pero que sigue mostrando que existen muchos elementos del procedimiento que requieren revisión y que pueden optimizarse.

Dado lo anterior, se torna necesario realizar investigaciones más amplias y hacer algunos cambios en el procedimiento con el fin de evitar que una terapia que se ha mostrado efectiva y eficaz siga siendo considerada como última opción y caiga en desuso.

Conflicto de intereses: Sin conflicto de intereses. 


\section{Bibliografía}

1. Mcdonald A, Walter G. The portrayal of ECT in American movies. J ECT. 2001; 17 (4): 264-74.

2. Walter G, Mcdonald A, Rey JM, Rosen A. Medical student knowledge and attitudes regarding ECT prior to and after viewing ECT scenes from movies. J ECT. 2002; 18 (1): 43-46.

3. Geddes J, Carney S, Cowen P, Goodwin G, Rogers R, Dearness $\mathrm{K}$ et al. Efficacy and safety of electroconvulsive therapy in depressive disorders: a systematic review and meta-analysis. Lancet. 2003; 361 (9360): 799-808.

4. Lauber C, Nordt C, Falcato L, Rössler W. Can a seizure help? The public's attitude toward electroconvulsive therapy. J Psychiatry Res. 2005; 134 (2): 205-209.

5. Rose D, Wykes T, Bindman J, Fleischmann P. Information, consent and perceived coercion: Patients' perspectives on electroconvulsive therapy. Br J Psychiatry. 2005; 186: 54-59.

6. Dowman J, Patel A, Rajput K. Electroconvulsive therapy attitudes and misconceptions. J ECT. 2005; 21 (2): 84-87.

7. McFarquhar T, Thompson J. Knowledge and attitudes regarding electroconvulsive therapy among medical students and the general public. J ECT. 2008; 24 (4): 244-253.

8. Buccelli C, Di Lorenzo P, Paternoster M, D'Urso G, Graziano $\mathrm{V}$, Niola M. Electroconvulsive therapy in Italy: will public controversies ever stop? J ECT. 2016; 32 (3): 207-211.

9. Guzmán-Sabogal Y, Acero-González Á, Romero-Tapia A, Ruiz-Moreno L, Tejada-Morales P. Prácticas, actitudes y conocimientos en relación a la terapia electroconvulsiva. Rev la Fac Med. 2018; 66 (3): 319-325.

10. Byrne P, Cassidy B, Higgins P, Rcsi FFA. Knowledge and attitudes toward electroconvulsive therapy among health care professionals and students. J ECT. 2006; 22 (2): 133-138.

11. Aki OE, Ak S, Sonmez PYE, Demir B. Knowledge of and attitudes toward electroconvulsive therapy among medical students, psychology students, and the general public. J ECT. 2013; 29 (1): 45-50.

12. Coalition Against Psychiatric Assault [Internet]. [cited 2019 Mar 3]. Available in: https://coalitionagainstpsychiatricassault. wordpress.com/

13. Wood JH, Chambers M, White SJ. Nurses' knowledge of and attitude to electroconvulsive therapy. J ECT. 2007; 23 (4): 251-254.

14. Teh S, Helmes E, Drake D. A Western Australian survey on public attitudes toward and knowledge of electroconvulsive therapy. Int J Soc Psychiatry. 2007; 53 (3): 247-271.

15. Sienaert P, De Becker T, Vansteelandt K, Demyttenaere K, Peuskens J. Patient satisfaction after electroconvulsive therapy. J ECT. 2005; 21 (4): 227-231.

16. Percepción. Definición de percepción. Diccionario de la lengua española. Edición del Tricentenario [Internet]. [cited 2019 Mar 6]. Disponible en: https://dle.rae.es/?id=SX9HJy3

17. Rajagopal R, Chakrabarti S, Grover S. Satisfaction with electroconvulsive therapy among patients and their relatives. J ECT. 2013; 29 (4): 283-290.

18. Chakrabarti S, Grover S, Rajagopal R. Perceptions and awareness of electroconvulsive therapy among patients and their families a review of the research from developing countries. J ECT. 2010; 26 (4): 317-322.

19. Health Knowledge, Attitudes, Practice-MeSH - NCBI [Internet]. [cited 2019 Feb 28]. Available in: https://www.ncbi.nlm.nih.gov/ mesh/?term = knowledge + health

20. Patient Satisfaction - MeSH - NCBI [Internet]. [cited 2019 Feb 28]. Available in: https://www.ncbi.nlm.nih.gov/mesh/68017060
21. Attitude - MeSH - NCBI [Internet]. [cited 2019 Feb 28]. Available in: https://www.ncbi.nlm.nih.gov/mesh/68001290

22. Malekian A, Amini Z. Knowledge of attitude toward experience and satisfaction with electroconvulsive therapy in a sample of Iranian patients. J ECT. 2009; 25 (2): 106-112.

23. Rajagopal R, Chakrabarti S, Grover S, Khehra N. Knowledge, experience \& attitudes concerning electroconvulsive therapy among patients \& their relatives. Indian J Med Res. 2012; 135: 201-210.

24. Flamarique I, Castro-fornieles J, Pons A, Bernardo M, Baeza I. Patients 'opinions about electroconvulsive therapy: what do adolescents with schizophrenia spectrum disorders think? J Child Adolesc Psychopharmacol. 2015; 25 (8): 641-648.

25. Dan A, Grover S, Chakrabarti S. Original article knowledge and attitude of patients with psychiatric disorders and their relatives toward electroconvulsive therapy. Indian J Psychol Med. 2014; 36 (3).

26. Grover SK, Chakrabarti S, Khehra N, Rajagopal R. Does the experience of electroconvulsive therapy improve awareness and perceptions of treatment among relatives of patients? J ECT. 2011; 27 (1): 67-72.

27. Kheiri M, Sahebalzamani M, Jahantigh M. The study of education effect on knowledge of, and attitudes toward electroconvulsive therapy among Iranian nurses and patients' relatives in a psychiatric hospital, 2009-2010. Procedia-Soc Behav Sci. 2011; 30: 256-260.

28. Bloch $\mathrm{Y}$, Linder $\mathrm{M}$, Kalman $\mathrm{N}$ et al. Adult and adolescent patient evaluations of electroconvulsive therapy in comparison to other therapeutic modalities. J ECT. 2018; 34 (1): 45-49.

29. Zhang Q, Zhou F, Zhang L, Ng CH, Ungvari GS, Wang G. Knowledge and attitudes of older psychiatric patients and their caregivers towards electroconvulsive therapy. J Japanese Psychogeriatrics Soc. 2018; 18 (5): 1-8.

30. Brown SK, Nowlin RB, Sartorelli R, Smith J, Johnson K. A retrospective review of clinical outcomes and satisfaction patient experience of electroconvulsive therapy. J ECT. 2018; 34 (4): 240-246.

31. Ross EL, Zivin K, Maixner DF. Cost-effectiveness of electroconvulsive therapy vs pharmacotherapy/psychotherapy for treatment-resistant depression in the United States. JAMA Psychiatry. 2018; 75 (7): 713-722.

32. Khalid N, Atkins M, Tredget J, Giles M, Champney-Smith K, Kirov G. The effectiveness of electroconvulsive therapy in treatmentresistant depression: A naturalistic study. J ECT. 2008; 24 (2): 141-145.

33. Price LH, Bertollo DN, Olfson M, Case BG, Marcus SC, Siegel CE et al. Declining use of electroconvulsive therapy in united states general hospitals. Biol Psychiatry. 2012; 73 (2): 119-126.

34. Freeman BCPL. ECT: I. Patients experiences and attitudes. Britain J Psychiatry. 1980; 137: 8-16.

35. Hughes J. Are patients shocked by ECT? J R Soc Med. 1981; 74 (7): 556-556.

36. Tang WK, Ungvari GS, Chan M, Grace W. Patients' and their relatives' knowledge of, experience with, attitude toward, and satisfaction with electroconvulsive therapy in Hong Kong, China. J ECT. 2002; 18 (4): 207-212.

37. Krahn LE, Rummans TA, Pileggi TS, Goodman JA, Smith GE. Patient satisfaction with electroconvulsive therapy. Mayo Clin Proc. 1999; 74 (10): 967-971.

38. Rajkumar AP, Saravanan B, Jacob KS. Perspectives of patients and relatives about electroconvulsive therapy: a qualitative study from Vellore, India. J ECT. 2006; 22 (4): 253-258.

39. Walter G, Koster K, Rey JM. Electroconvulsive therapy in adolescents: experience, knowledge, and attitudes of recipients. J Am Acad Child Adolesc Psychiatry. 1999; 38 (5): 594-599. 
40. Taieb O, Flament MF, Corcos M, Jeammet P, Basquin M, Mazet $\mathrm{P}$ et al. Electroconvulsive therapy in adolescents with mood disorder: patients' and parents' attitudes Olivier. Psychiatry Res. 2001; 104: 183-190.

41. Jelovac A, O'Connor S, McCarron S, McLoughlin DM. Autobiographical memory specificity in major depression treated with electroconvulsive therapy. J ECT. 2016; 32 (1): 38-43.

\section{Correspondencia:}

\section{Ángela Rocío Acero González}

Campus del Puente del Común,

Km. 7, Autopista Norte de Bogotá Chía,

Cundinamarca,Colombia Edificio $\mathrm{H}$, Oficina

205H.

Tel: 5718615555 , ext. 23022.

E-mail: angela.acero@unisabana.edu.co 